\title{
Hippocampal electrophysiological responses and changes in oxidative stress marker and serum lipid profile to pharmacological and non-pharmacological treatments of high-fat-fructose diet induced metabolic syndrome
}

Abstract The aim of our study was to evaluate the possibility of influencing the risk factors of metabolic syndrome (MetS) and metabolic cognitive syndrome. As a model of MetS, we used high-fat-fructose diet (HFFD) fed hypertriacylglycerolemic (HTG) rats. Control group included HTG rats fed with HFFD during 8 weeks (HFFD8). Furthermore, we tested the effect of pharmacological and non-pharmacological therapies. Non-pharmacological therapy, which we chose, was a change in diet from HFFD (5 weeks) to standard one (3 weeks) and thus caloric restriction (HFFD5+3). The drug we used was rosmarinic acid (RA; 100mg/kg), which we administered to rats after 5 weeks of HFFD once a day for consecutive 3 weeks with current change in diet to standard one (HFFD5+3+RA) or during lasting last 3 weeks of HFFD (HFFD8+RA). After 8 weeks of experiment, lipid peroxidation markers, lipid profile of blood serum, and neuronal transmission and synaptic plasticity (long-term potentiation [LTP]) in hippocampal sections were evaluated in vitro. We observed a significant effect of dietary change in lipid profile (decreased total cholesterol and lowdensity lipoprotein cholesterol [LDL-cholesterol] and increased high-density lipoprotein cholesterol [HDL-cholesterol]). The combination of pharmacological and non-pharmacological treatments caused a decrease in total cholesterol, LDL-cholesterol, and lipid peroxidation in blood serum. Change in HFFD to standard diet without treatment resulted in slight improvement in neuronal transmission in the hippocampus and caloric restriction alone also had positive effect on LTP maintenance. Our results suggest that combination of pharmacological and non-pharmacological approaches had better impact on the biochemical parameters of MetS in blood serum, but weak impact on neuronal functions in the hippocampus, where the expected positive effect was achieved only by caloric restriction.

Keywords metabolic syndrome-high-fat-fructose diet-hippocampus-cholesterol-oxidative stress-rosmarinic acid-caloric restriction

\section{INTRODUCTION}

Metabolic cognitive syndrome is a condition when metabolic syndrome (MetS) can lead to deterioration of cognitive abilities. Cognitive skills include memory, spatial memory, learning, executive functions, attention, language skills (Frisardi et al., 2010). MetS parameters such as obesity, dyslipidemia, hypertension, insulin resistance, inflammation, and oxidative stress may cause changes in the physiology that can affect cognition. The mechanisms responsible for this link are not clear. Mechanisms associated with systemic inflammation and inflammation in the nervous system, insulin resistance and oxidative stress in the brain, atherosclerosis, endothelial impairment and impaired capillary reactivity in the central nervous system, and abnormal lipid metabolism in the brain are discussed (Stoeckel et al., 2016). Rosmarinic acid (RA) is a phenolic acid that is contained in several plants used in medicine, mainly belonging to the Laminaceae family. It has several physiological effects, such as antioxidant, anti-inflammatory, antiviral, antibacterial, antidepressant, anticarcinogenic, chemopreventive, and neuroprotective effects (Bhatt at al. 2013). RA passes the blood-brain barrier through receptor-mediated transfer, combined with the ligand CRM197. RA then easily enters brain endothelial cells by endocytosis (Kuo and Rajesh, 2017). Recently, it was found that non-pharmacological approach such as changing diet 
habits or caloric restriction can reduce insulin resistance and increase physical fitness and overall metabolic health, which appears to reduce the risk for cardiovascular diseases (Grundy et al., 2013).

\section{MATERIAL AND METHODS}

\section{Experimental design}

We used Prague hereditary hypertriacylglycerolemic (HTG) rats from the Department of Toxicology and Breeding of Laboratory Animals of the Institute of Pharmacology and Toxicology, Centre of Experimental Medicine, Slovak Academy of Sciences (IEPT CEM SAS), Dobrá Voda. The experiments were approved by the State Veterinary and Food Administration of the Slovak Republic and the Ethical Committee of IEPT CEM SAS. At the beginning of the experiment, the animals were 10 weeks old. The animals had ad libitum access to food and water and light cycle 12/12 (12 hours dark, 12 hours light). We divided the animals into four groups: HFFD8 group: HTG rats were fed 8 weeks with modified diet (high-fat-fructose diet; HFFD); HFFD5+3 group: HTG rats were fed 5 weeks with HFFD and then 3 weeks with standard diet; HFFD8+RA group: HTG rats were fed 8 weeks with HFFD and last 3 weeks RA ( $100 \mathrm{mg} /$ $\mathrm{kg}$ ) was administered to them; HFFD5+3+RA group: HTG rats were fed 5 weeks with HFFD and then 3 weeks with standard diet along with the administration of RA ( $100 \mathrm{mg} / \mathrm{kg})$. RA was administered once a day on crackers. Pellets of modified diet contained $1 \%$ cholesterol, $7.5 \%$ pork lard, and $10 \%$ fructose.

\section{Determination of oxidative damage}

Malondialdehyde (MDA), as marker of lipid peroxidation, was determined by the double heating method of Drapper and Hadley (1990). The principle of the method was spectrophotometric measurement of solution staining during the reaction of tertiary butyl alcohol with MDA.

\section{Determination of lipid profile}

Erba Lachema Ltd (Brno, Czech Republic) kits were used to determine the lipid profile from the blood serum. We measured the levels of total cholesterol, low-density lipoprotein cholesterol (LDL-cholesterol), and high-density lipoprotein cholesterol (HDL-cholesterol). Absorbance of the resulting colored compound was measured spectrophotometrically.

\section{Electrophysiological measurement of neuronal function in rat hippocampus in vitro}

Neurotransmission was determined by recording and digitizing electrically induced responses of hippocampus. We used artificial cerebrospinal fluid (ACSF) composed of $124 \mathrm{mmol} / \mathrm{I}$ of $\mathrm{NaCl}, 3.3 \mathrm{mmol} / \mathrm{l}$ of $\mathrm{KCl}, 1.25 \mathrm{mmol} / \mathrm{l}$ of $\mathrm{KH}_{2} \mathrm{PO}_{4^{\prime}}, 2.4 \mathrm{mmol} / \mathrm{l}$ of $\mathrm{MgSO}_{4^{\prime}} 2.5 \mathrm{mmol} / \mathrm{l}$ of CaCl $2^{\prime}, 26 \mathrm{mmol} / \mathrm{I}$ of $\mathrm{NaHCO}_{3}$, and $10 \mathrm{mmol} / \mathrm{l}$ of glucose and saturated with $95 \% \mathrm{O}_{2}+5 \% \mathrm{CO}_{2}$ at a pH of 7.4. Hippocampal slices (400$\mu \mathrm{m}$ thick) were stimulated by bipolar stainless steel wire electrode. Electrically evoked responses were recorded using glass microelectrode filled with ACSF (3-5 M $\Omega$ ) in the stratum radiatum of the rat hippocampus. We assessed the amplitude of excitatory postsynaptic potential (EPSP) as a measure of neuronal transmission. Long-term potentiation (LTP) was elicited by high-frequency stimulation $(100 \mathrm{~Hz}, 1 \mathrm{~s})$ and recordings continued next 40 min after train induction.

\section{Statistical evaluation}

The data were statistically evaluated using the GraphPad Prism6 Software (GraphPad, La Jolla, USA). Data were expressed as means \pm standard error of the mean (SEM). One-way analysis of variance (ANOVA) was used to evaluate the difference among all experimental groups (using the Bonferroni multiple comparison test). The limit of $p<0.05$ was considered as statistically significant difference.

\section{RESULTS}

Total cholesterol increased significantly in all groups after 5 weeks of HFFD. Changing the diet to standard caused a decrease in its levels, which is comparable to the pre-treatment values. HDL-cholesterol decreased after consumption of HFFD diet and had a tendency to increase again as a result of both pharmacological and non-pharmacological approaches as well as their combination, but the combination did not produce a further increase in the effect. LDL-cholesterol levels increased after 5 weeks of HFFD and decreased after changing diet as well as decreased in the HFFD5+3+RA group (Table 1).

Lipid peroxidation was determined by the increase in MDA in blood serum after 5 -week consumption of HFFD in all 4 groups. The combination of pharmacological and nonpharmacological therapies resulted in a decrease in lipid peroxidation, but the values were still elevated compared to the original ones. A change in diet or RA treatment alone did not cause a significant improvement (Figure 1A). No change in lipid peroxidation was observed in the cortex (Figure 1B). During the recordings of electrically evoked responses in hippocampal slices in vitro, we found that diet change alone caused tendency to increase the amplitude of EPSP, (Figure 2A). LTP was damaged in HFFD8, HFFD+RA, and HFFD5+3+RA groups, whereas it persisted (134 $\pm 22 \%$ ) in hippocampus of rats whose diet was changed to standard (Figure $2 \mathrm{~B}$ ).

\section{DISCUSSION}

MetS is a cluster of risk factors such as obesity, dyslipidemia, hypertension, oxidative stress, inflammation, elevated glucose levels, or insulin resistance. These metabolic disturbances can cause cognitive decline throughout several 
Table 1: Cholesterol profile in blood serum of rats ( $\mathrm{mmol} / \mathrm{l})$.

HFFD, high-fat-fructose diet; HFFD8, HTG rats fed 8 weeks with HFFD; HFFD5+3, HTG rats fed 5 weeks with HFFD and then 3 weeks with standard diet; HFFD8+RA, HTG rats fed 8 weeks with HFFD and during last 3 weeks of this diet treated with rosmarinic acid; HFFD 5+3+RA, HTG rats fed 5 weeks with HFFD and then 3 weeks with standard diet and treated with rosmarinic acid. Data are expressed as means $\pm S E M ; n=10 .{ }^{*} p<0.05$ versus same group before the diet, ${ }^{* *} p<0.01$ versus same group before the diet, ${ }^{* * *} p<0.001$ versus same group before the diet, ${ }^{*} p<0.05$ versus same group after 5 weeks of diet,,$" * 0.01$ versus same group after 5 weeks of diet, ${ }^{\# \#} p<0.001$ versus same group after 5 weeks of diet, ap $<0.05$ versus HFFD 8 at the same time, bp<0.05 versus HFFD8+RA at the same time.

\begin{tabular}{|c|c|c|c|c|c|c|c|c|c|}
\hline & \multicolumn{3}{|c|}{ Total cholesterol } & \multicolumn{3}{|c|}{ HDL-cholestero } & \multicolumn{3}{|c|}{ LDL-cholesterol } \\
\hline & $\begin{array}{c}\text { Before } \\
\text { diet }\end{array}$ & $\begin{array}{l}\text { After } 5 \\
\text { week }\end{array}$ & After diet & $\begin{array}{c}\text { Before } \\
\text { diet }\end{array}$ & $\begin{array}{c}\text { After } 5 \\
\text { week }\end{array}$ & After diet & $\begin{array}{c}\text { Before } \\
\text { diet }\end{array}$ & $\begin{array}{c}\text { After } 5 \\
\text { week }\end{array}$ & After diet \\
\hline HFFD 8 & $\begin{array}{l}1.84 \pm \\
0.05\end{array}$ & $\begin{array}{c}2.62 \pm \\
0.08^{* * *}\end{array}$ & $\begin{array}{c}3.13 \pm \\
0.11^{* * * *}\end{array}$ & $\begin{array}{c}0.78 \pm \\
0.03\end{array}$ & $\begin{array}{c}0.50 \pm \\
0.03^{* * * *}\end{array}$ & $\begin{array}{l}0.61 \pm \\
0.01^{*}\end{array}$ & $\begin{array}{c}0.40 \pm \\
0.02\end{array}$ & $\begin{array}{c}1.19 \pm \\
0.05^{* * * *}\end{array}$ & $\begin{array}{c}1.53 \pm \\
0.09^{* * * * \# \#}\end{array}$ \\
\hline $\begin{array}{l}\text { HFFD } \\
5+3\end{array}$ & $\begin{array}{c}1.76 \pm \\
0.04\end{array}$ & $\begin{array}{l}2.92 \pm \\
0.11^{* * * *}\end{array}$ & $\begin{array}{c}2.19 \pm \\
0.04 \# \# \# \text { ab }\end{array}$ & $\begin{array}{c}0.77 \pm \\
0.05\end{array}$ & $\begin{array}{c}0.33 \pm \\
0.01^{* * *}\end{array}$ & $\begin{array}{c}0.50 \pm \\
0.04^{* * * *}\end{array}$ & $\begin{array}{c}0.38 \pm \\
0.02\end{array}$ & $\begin{array}{c}1.06 \pm \\
0.09^{* * * *}\end{array}$ & $\begin{array}{c}0.60 \pm \\
0.09 \# \# \# \text { ab }\end{array}$ \\
\hline $\begin{array}{l}\text { HFFD } \\
8+R A\end{array}$ & $\begin{array}{c}1.99 \pm \\
0.04\end{array}$ & $\begin{array}{c}2.78 \pm \\
0.16^{* * * *}\end{array}$ & $\begin{array}{c}2.98 \pm \\
0.09^{* * * *}\end{array}$ & $\begin{array}{c}0.84 \pm \\
0.02\end{array}$ & $\begin{array}{c}0.46 \pm \\
0.02^{* * *}\end{array}$ & $\begin{array}{c}0.54 \pm \\
0.03^{* * *}\end{array}$ & $\begin{array}{c}0.37 \pm \\
0.03\end{array}$ & $\begin{array}{c}1.11 \pm \\
0.06^{* * *}\end{array}$ & $\begin{array}{c}1.41 \pm \\
0.05^{* * * \#}\end{array}$ \\
\hline $\begin{array}{c}\text { HFFD } \\
5+3+\text { RA }\end{array}$ & $\begin{array}{c}1.99 \pm \\
0.08\end{array}$ & $\begin{array}{l}2.70 \pm \\
0.13^{* * *}\end{array}$ & $\begin{array}{c}2.23 \pm \\
0.07 \# \# \# \text { ab }\end{array}$ & $\begin{array}{c}0.83 \pm \\
0.03\end{array}$ & $\begin{array}{c}0.53 \pm \\
0.02^{* * *}\end{array}$ & $\begin{array}{l}0.64 \pm \\
0.04^{* *}\end{array}$ & $\begin{array}{c}0.33 \pm \\
0.02\end{array}$ & $\begin{array}{c}1.26 \pm \\
0.06^{* * *}\end{array}$ & $\begin{array}{c}0.57 \pm \\
0.05 \# \# \# \text { ab }\end{array}$ \\
\hline
\end{tabular}
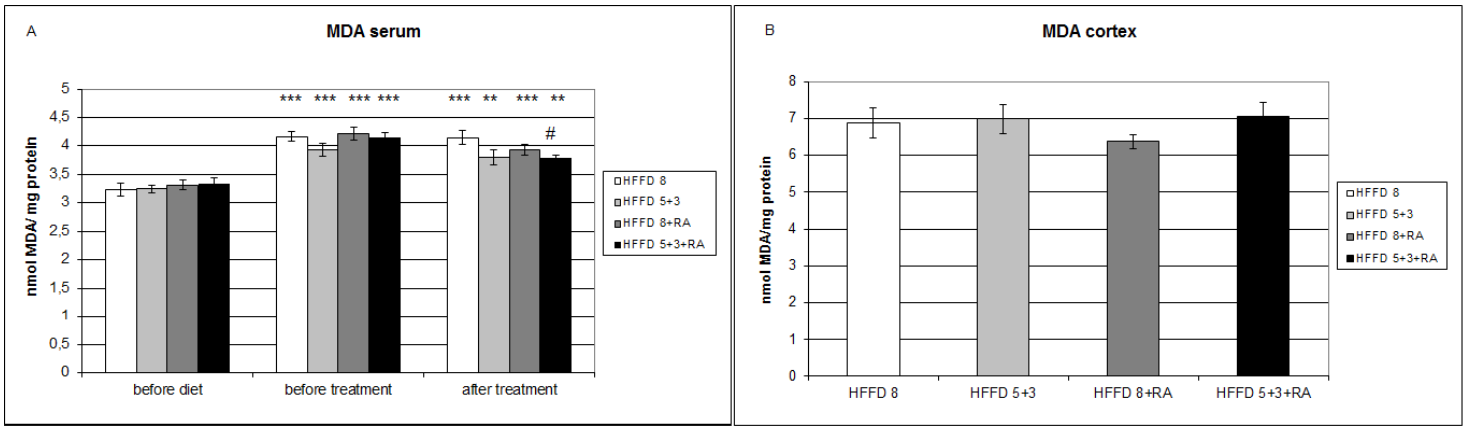

Figure 1: Markers of lipid peroxidation in (A) blood serum and (B) cortex (mmol/mg protein). HFFD, high-fat-fructose diet; HFFD8, HTG rats fed 8 weeks with HFFD; HFFD5+3, HTG rats fed 5 weeks with HFFD and then 3 weeks with standard diet; HFFD8+RA, HTG rats fed 8 weeks with HFFD and during last 3 weeks of this diet treated with rosmarinic acid; HFFD5+3+RA, HTG rats fed 5 weeks with HFFD and then 3 weeks with standard diet and treated with rosmarinic acid; MDA, malondialdehyde. Data are expressed as means \pm SEM; $n=10$. ${ }^{* *} p<0.01$ versus same group before the diet, ${ }^{* * *} p<0.001$ versus same group before the diet, ${ }^{*} p<0.05$ versus same group after 5 weeks of diet.
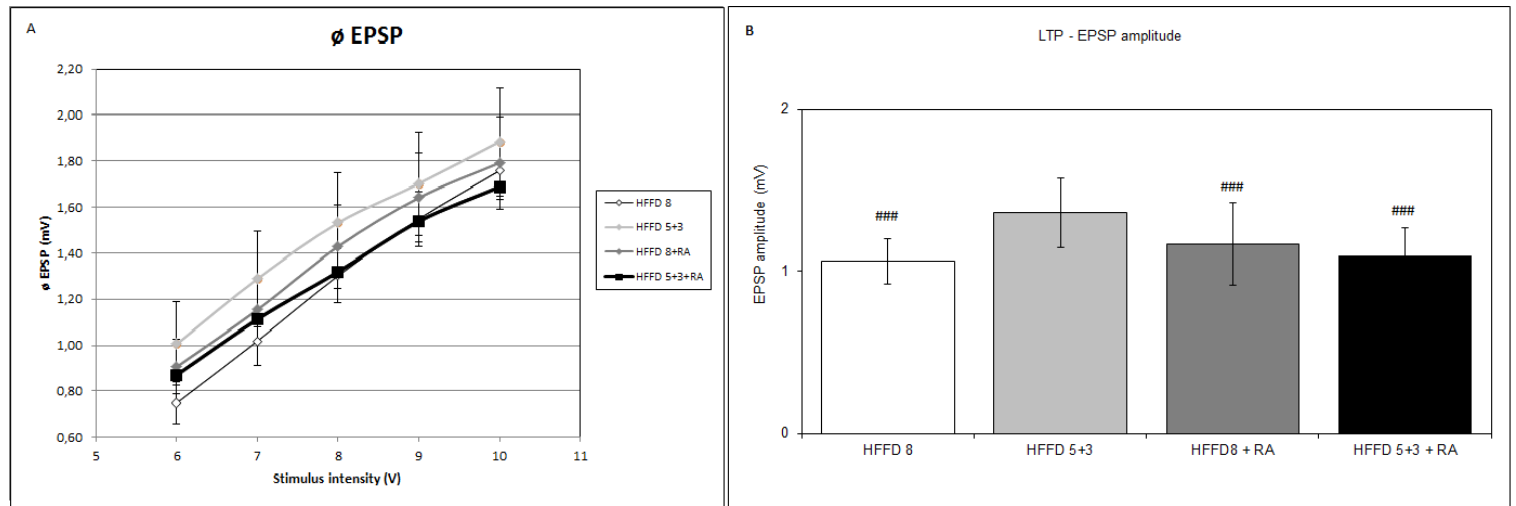

Figure 2: Electrically induced responses from hippocampal slices expressed as (A) excitatory postsynaptic potential (EPSP) amplitude $(\mathrm{mV})$ (stimulus intensity 6-10 V) and (B) Long-term potentiation (LTP) (normalized values). HFFD, high-fat-fructose diet; HFFD8, HTG rats fed 8 weeks with HFFD; HFFD5+3, HTG rats fed 5 weeks with HFFD and then 3 weeks with standard diet; HFFD8+RA, HTG rats fed 8 weeks with HFFD and during last 3 weeks of this diet treated with rosmarinic acid; HFFD 5+3+RA, HTG rats fed 5 weeks with HFFD and then 3 weeks with standard diet and treated with rosmarinic acid. Data are expressed as means \pm SEM; for (a) $n=17-24$ hippocampal slices/10 rats/group; and for (b) $n=10$ hippocampal slices $/ 10$ rats/group. ${ }^{\# \# ~} p<0.001$ versus HFFD $5+3$. 
mechanisms. Our animal model was a combination of geneticand diet-induced MetS-like conditions. Metabolic changes of MetS can lead to the development of cardiovascular and cerebrovascular diseases, and it is, therefore, necessary to look for an appropriate method of intervention. In the present work, we tested the effect of pharmacological and non-pharmacological treatments and their combination on individual risk factors. Changing diet to standard caused decrease in total cholesterol levels almost to their original levels. HDL-cholesterol tended to rise again as a result of both pharmacological and non-pharmacological therapies. LDL-cholesterol levels were increased because of HFFD and decreased after caloric restriction. Changing the diet to standard reduced lipid peroxidation, and RA has multiplied this effect, but RA alone had no effect. No change in the oxidative stress marker MDA was found in rat brain cortex. Neuronal transmission was slightly improved in hippocampus by dietary change as well as LTP was retained in this group, but RA alone or its combination with caloric restriction did not cause any improvement in hippocampal function.

In our study, we used three ways to ameliorate MetS components, that is, caloric restriction induced by changing HFFD to standard diet, pharmacological treatment with RA, and combination of both approaches. Caloric restriction is an effective therapeutic approach to improve metabolic state in the body. Caloric restriction associated weight loss decreases the accumulation of triacylglycerols (TAG) in tissues. It also causes decrease in adipose mass, oxidative stress, and inflammation, leading normalization of glucose homeostasis and lipid metabolism (Nikhra, 2018). RA has several biological effects, such as antiviral, antibacterial, anti-inflammatory, antioxidant, and anticancer effects (Pettersen and Simmons, 2003). RA promotes good endothelial and blood cell status and is also used to combat skin carcinogenicity as it can be absorbed through and stored in the skin, muscle, and bones (Osakabe et al., 2004; Ueda et al., 2003). It is also absorbed from the gastrointestinal tract; thus we administered it orally (Al-Sereiti, 1999). Unconjugated RA remains in the bloodstream long enough to reach the brain (Fale et al., 2011) and it crosses the blood-brain barrier via transporters (Kuo and Rajesh, 2017). On the basis of the literary data, effect of RA has not been studied in combination with caloric restriction so far.

We found that lipid peroxidation in blood serum increased after consumption of HFFD. The combination of pharmacological and non-pharmacological therapies caused a significant decrease in lipid peroxidation, but the values were still elevated compared to the original ones. A change in diet or RA alone did not cause a significant change. No change in lipid peroxidation was observed in the cortex. Matsuo and coauthors (1993) found that caloric restriction reduces the rate of accumulation of oxidatively damaged molecules and inhibits the increase in lipid peroxidation. It has been repeatedly reported that caloric restriction is capable of inducing mechanisms to protect against stress, especially those involved in the detoxification of reactive oxygen species (ROS) (Ristow and Schmeisser, 2014). RA has the ability to penetrate lipid bilayer, which alters membrane fluidity and protects cell membrane against chain-breaking free radicals (Fadel et al., 2011).

We found out that total cholesterol levels were increased after HFFD. Thus caloric restriction in combination with changing HFFD to standard diet plus RA treatment caused a decrease in these levels. HDL-cholesterol levels decreased after the consumption of food rich in fat and fructose. Significant improvement in HDL-cholesterol levels was achieved by 3-week caloric restriction applied after HFFD. LDL-cholesterol levels increased after HFFD and decreased after caloric restriction and also in the combined HFFD5+3+RA group. LDL-cholesterol is now considered as MetS sign, and it is still the most common marker of cardiovascular diseases (CVD) risk and atherogenic dyslipidemia. The results of a clinical study by Al-Sarraj and co-workers (2009) showed a significant decrease in small LDL-cholesterol particles in individuals who consumed less calories, in particular less saccharides. Fat reduction in the diet has minimal effect on $\mathrm{HDL}$-cholesterol but carbohydrate restriction had a significant effect (Al Sarraj et al., 2009). The mechanism that may be responsible for the improvement in the lipid profile after caloric restriction, especially carbohydrate restriction, is that high insulin suppresses lipolysis and increases de novo lipogenesis. Caloric limitation simultaneously leads to a decrease in the concentration of malonyl-coenzyme $A$ and dis-inhibition of carnitine acyltransferase, which caused an increase in $\beta$-oxidation of fatty acids (Volek et al., 2009). Our results are in accordance with these results and also with the results of Govindaraj and Pillai (2015), who reported elevated levels of free fatty acids, total cholesterol, and TAG in the rats with HFD. Rats fed with HFD have hypertriacylglycerolemia and hypercholesterolemia, the condition which is reversed after oral administration of RA. These results show the beneficial effects of RA in preventing metabolic complications.

In our work, we anticipated an improvement in the electrophysiological responses of the hippocampus. In the electrophysiological response of the hippocampus, we observed that diet change alone caused a slight increase in the EPSP amplitude. No improvement in neurotransmission was observed in RA-treated group and in combination of non-pharmacological and pharmacological treatments. LTP retained only in the group with caloric restriction. Recently, it was shown that caloric restriction induces a neuroendocrine response such as an increase in neuropeptide Y (NPY) (Minor et al., 2009; Bi et al., 2003). Activation of NPY receptors has neuroprotective effects in various regions of the brain and results in delayed neurodegenerative damage (Decressac and Barker, 2012). In addition to NPY, the production of ghrelin, which has many physiological functions throughout the body and in the central nervous system, is also increasing 
by caloric restriction (Ferrini et al., 2009). Ghrelin is also involved in memory and learning and has a neuroprotective effect in neurodegenerative diseases and in ischemic brain damage (Spencer et al., 2014). Previous studies, as well as our work, suggest that cognitive decline occurs with MetS, which deepens over time. Among the factors connecting MetS with cognitive decline, the brain-derived neurotrophic factor (BDNF) in the hippocampus is reduced. After 28 day of caloric restriction, body weight, insulin and fasting glucose levels, adiponectin, systolic blood pressure, and oxidative stress in the hippocampus were significantly reduced and BDNF expression in the hippocampus was significantly higher (Kishi et al., 2015). We assume that in mechanism of slightly improved response in the hippocampus on electrical stimulation induced by caloric restriction, an increase in BDNF expression and its ability to affect neurotransmitter release might be involved (Sasaki et al., 2013; Yan and Yan, 2006). Concerning RA effect, it was reported that glutamate receptor-2 (GluR2 ) is enhanced by RA treatment, which has an important impact on synaptic plasticity because GluR-2 has an effect on intracellular translocation and folding of a-amino-3-hydroxy5-methyl-4-isoxazolepropionic acid (AMPA) receptor subunits (Cull-Candy et al., 2006). RA also acts at the level of N-Methyld-aspartate (NMDA) receptors (Morris, 1989). The reason or mechanism why RA did not act in our study on improvement of hippocampal neurotransmission as well as LTP is unclear. There is a possibility that administration of RA was not long enough or there was some underlying mechanism that inhibited passing of RA through blood-brain barrier in our experimental design. On the other hand, the best reduction of blood serum MDA level was achieved by the combination of RA treatment and caloric restriction. As a conclusion, we can assume that caloric restriction, RA acid treatment, and their combination could be promising in the management of MetS-related disorders; therefore, further detailed studies are needed.

\section{ACKNOWLEDGMENTS}

The study was supported by the Slovak Grant Agency for Science with grants VEGA 2/0054/15 (2015 -2018) and VEGA 2/0120/19 (2019-2022).

\section{ABBREVIATIONS}

ACSF - artificial cerebrospinal fluid

AMPA - a-amino-3-hydroxy-5-methyl-4-isoxazolepropionic acid

ANOVA - analysis of variance

BDNF - brain-derived neurotrophic factor

CVD - cardiovascular diseases

EPSP - excitatory postsynaptic potential

GluR 2 - glutamate receptor 2

HDL-cholesterol - high-density lipoprotein cholesterol

HFD - high-fat diet

HFFD - high-fat-fructose diet

HTG - hypertriacylglycerolemic

IEPT CEM SAS - Institute of Experimental Pharmacology and Toxicology, Centre of Experimental Medicine, Slovak Academy of Sciences

LDL-cholesterol - low-density lipoprotein cholesterol

MDA - malondialdehyde

MetS - metabolic syndrome

$n$ - number

NMDA - N-Methyl-d-aspartate

NPY - neuropeptide $Y$

RA - rosmarinic acid

ROS - reactive oxygen species

SEM - standard error of the mean

TAG - triacylglycerols

\section{References}

[1] Al-Sarraj T, Saadi H, Volek JS, Fernandez ML: Carbohydrate restriction favorably alters lipoprotein metabolism in Emirati subjects classified with the metabolic syndrome. Nutr Metab Cardiovasc Dis. 2010,20(10):720-726.

[2] Al-Sereiti MR, Abu-Amer KM, Sen P: Pharmacology of Rosemary (Rosmarinus officinalis Linn.) and Its Therapeutic Potentials. Indian J Exp Biol. 1999, 37:124-130.

[3] Bhatt R, Mishra N, Bansal PK: Phytochemical, pharmacological and pharmacokinetics effects of rosmarinic acid., JPSI. 2013, 2(2):28-34.

[4] Bi S, Robinson BM and Moran TH. Acute food deprivation and chronic food restriction differentially affect hypothalamic NPY mRNA expression. Am J Physiol Regul Integr Comp Physiol 2003, 285:1030-36.

[5] Cull-Candy S, Kelly L, Farrant M: Regulation of Ca2p-permeable AMPA receptors: synaptic plasticity and beyond, Curr. Opin. Neurobiol. 2006, 16:288-297.
[6] Decressac M, Barker RA. Neuropeptide $Y$ and its role in CNS disease and repair. Exp Neurol. 2012, 238:265-272.

[7] Drapper HH, Hadley M: Malondialdehyde determination as index of lipid peroxidation. Methods Enzymol. 1990, 186:421-431.

[8] Fadel O, El Kirat K, Morandat S: The natural antioxidant rosmarinic acid spontaneously penetrates membranes to inhibit lipid peroxidation in situ. Biochim Biophys Acta. 2011, 1808:29732980.

[9] Fale PLV, Madeira PJA, Florencio MH, Ascensao L, Serralheiro MLM: Function of Plectranthus barbatus herbal tea as neuronal acetylcholinesterase inhibitor. Food \& Function. 2011; 2: 130-136.

[10] Ferrini F, Salio C, Lossi L, Merighi A. Ghrelin in central neurons. Curr Neuropharmacol. 2009, 7:37-49.

[11] Frisardi V, Solfrizzi V, Seripa D, Capurso C, Santamato A, Sancarlo D, Vendemiale G, Pilotto A, Panza F: Metabolic-cognitive syndrome: a cross-talk between metabolic syndrome and Alzheimer's disease. Ageing Res Rev 1. 2010; 9:399- 417. 
[12] Govindaraj J, Sorimuthu Pillai S: Rosmarinic acid modulates the antioxidant status and protects pancreatic tissues from glucolipotoxicity mediated oxidative stress in high-fat diet: Streptozotocin-induced diabetic rats. Mol Cell Biochem. 2015, 404:143- 159.

[13] Grundy SM, Barlow CE, Farrell SW, Vega GL, Haskell WL: Cardiorespiratory fitness and metabolic risk. Am J Cardiol, 2012, 109:988-993.

[14] Kishi T, Hiroka Y, Nagayama T, Isegawa K, Katsuki M, Takesue K, Sungawa K: Calorie restriction improves cognitive decline via upregulation of brain-derived neurotrophic factor. Int Heart J. 2014, 56(1):110-115.

[15] Kuo Y-Ch, Rajesh R:Targeted delivery of rosmarinic acid across the blood-brain barrier for neuronal rescue using polyacrylamidechitosan-poly(lactide-co-glycolide) nanoparticles with surface cross-reacting material 197 and apolipoprotein E. Int J Pharm. 2017, 528:228-241.

[16] Matsuo M, Gomi F, Kuramoto K, Sagai M: Food restriction suppresses an age-dependent increase in the exhalation rate of pentane from rats: a longitudinal study. J. Gerontol. 1993, 48(4):B133-136.

[17] Minor RK, Chang JW, de Cabo R: Hungry for life: How the arcuate nucleus and neuropeptide $Y$ may play a critical role in mediating the benefits of calorie restriction. Mol Cell Endocrinol. 2009, 299:79-88.

[18] Morris RG: Synaptic plasticity and learning: selective impairment of learning rats and blockade of long-term potentiation in vivo by the N-methyl-D-aspartate receptor antagonist AP5, J Neurosci. 1989, 9:3040-3057.

[19] Nikhra V: Revisiting Caloric Restriction as Therapeutic Strategy for Metabolic Syndrome, T2DM and Obesity. IOD. 2018, 2(3):1-5.

[20] Osakabe N, Yasuda A, Natsume M, Yoshikawa T: Rosmarinic acid inhibits epidermal inflammatory responses: anticarcinogenic effect of Perilla frutescens extract in the murine two-stage skin model. Carcinogenesis. 2004, 25:549-557.

[21] Pettersen M, Simmonds MSJ: Rosmarinic acid. Phytochemistry. 2013, 12:207-227.

[22] Ristow M, Schmeisser K: Mitohormesis: promoting health and lifespan by increased levels of reactive oxygen species (ROS). Dose Response. 2014, 12(2):288-341.

[23] Sasaki K, El Omri A, Kondo S, Han J, Isoda H: Rosmarinus officinalis polyphenols produce anti-depressant like effect through monoaminergic and cholinergic functions modulation, Behav Brain Res. 2013, 238:86-94.

[24] Spencer SJ, Miller AA, Andrews ZB. The role of ghrelin in neuroprotection after ischemic brain injury. Brain Sci. 2013, 3:344-59.

[25] Stoeckel LE, Arvanitakis Z, Gandy S, Small D, Pascual-Leone A, Pawlyk A, Shewrin R, Smith P: Complex mechanisms linking neurocognitive dysfunction to insulin resistance and other metabolic dysfunction. Version 2. F1000Res. 2016; 5:353.
[26] Ueda H, Yamazaki C, Yamazaki M: Inhibitory effect of Perilla leaf extract and luteolin on mouse skin tumor promotion. Biol Pharm Bull. 2003; 26:560-563.

[27] Volek JS, Phinney SD, Forsythe CE, Quann EE, Wood RJ, Puglisi MJ, Kreamer WJ, Bibus DM, Fernandez ML, Feinman RD: Carbohydrate restriction has a more favorable impact on the metabolic syndrome than a low fat diet. Lipids. 2009, 44:297-309.

[28] Yan FX, Yan T: Effect of rosmarinic acid against the PC12 cell injures induced by glutamate, Acta Pharmacol. Sin. 2006, 27:100. 